\title{
Comparative Evaluation of Completeness of Traditional Narrative versus Electronic Synoptic Operative Reports for Ovarian, Peritoneal and Fallopian Tube Cancer Surgery in a Tertiary Government Hospital
}

\author{
Aubrey Y. Señeris, MD and Jean Anne B. Toral, MD, MSc \\ Division of Gynecologic Oncology, Department of Obstetrics and Gynecology, Philippine General Hospital, University of the Philippines Manila
}

\begin{abstract}
Objective. This study aimed to compare the completeness and ease of use of narrative reports (NR) submitted by residents compared to electronic synoptic reports (SR) by gynecologic oncology fellows for patients who underwent ovarian, fallopian, and peritoneal cancer surgery.
\end{abstract}

Methods. We conducted a cross-sectional study in the Department of Obstetrics-Gynecology of the Philippine General Hospital from August to November 2019. We assessed the NRs and electronic SRs for completeness of data using quality indicators.

Results. The average percentage of completeness of quality indicators is $77.1 \%(35.7 / 65)$. Eight indicators were absent in all NRs. Reporting of residual lesions was low (29.1\%). The mean time to accomplish SRs (10.4 minutes) was significantly shorter than the mean time to accomplish NRs (21.9 minutes) ( $p$ value $=0.0001$ ). SRs were assessed to be superior to NRs in several areas of surgery for quality, completeness and timeliness.

Conclusion. This study showed that the NRs should be improved and periodic audit must be done to maintain quality assurance. The use of SR appears to be favorable and superior in terms of time required to accomplish.

Key Words: operative record, synoptic report, narrative report

\section{INTRODUCTION}

Quality improvement is an important component of the health care system. At present, few quality indicators are available that measure the process of care that occur during gynecologic surgery. Measuring the completeness of the operative report is a potential area for quality improvement. The elements contained in the operative report are potential sources for data quality indicators. However, little is known about the completeness of the gynecologic operative reports.

The operative report is a vital medicolegal document accomplished by the surgeon at the end of the surgical procedure. It serves as an important source of information

Corresponding author: Aubrey Y. Señeris, MD

Division of Gynecologic Oncology

Department of Obstetrics and Gynecology

Philippine General Hospital

University of the Philippines Manila

Taft Avenue, Manila 1000, Philippines

Email: aubreyseneris@gmail.com for other health-care providers involved in subsequent care such as reimbursement proceedings, follow-up care by nursing staff, therapists, and physician, especially for referrals to subspecialty clinics, such as the Section of Gynecologic Oncology. It could also be a valuable tool for surgical training. 
The traditional narrative operative report (NR) refers to the handwritten or a typewritten document in a narrative format describing the indication, procedures, intraoperative findings and complications of the surgery. There is no specific training during obstetrics and gynecology residency programs to prepare such documentation. As a consequence, operative reports contain non-standardized information and have been an identified area of weakness in gynecologic surgery.

Synoptic operative report (SR) refers to the use of a computerized, procedure-specific, template-based and standardized record of the operation. With the development of electronic health records, templates are being adopted rapidly by the medical communities worldwide. Electronic synoptic operative reports ensure documentation of all critical components and findings during complex surgical procedures.

Little is known about how closely operative reports reflect what was actually performed during an operation and the intraoperative findings in a gynecological procedures. Studies that aim to clarify the objectives of those reports and improve their efficacy are lacking. Published studies suggest that the $\mathrm{SR}$ is superior than the NR by the surgeon. It ensures that pertinent information are recorded in a standardized way and documents can be immediately generated for use.

To the author's knowledge, there are no local studies evaluating the completeness of a NR and the role of an electronic SR in the training of gynecologic residents and its potential to improve the accuracy, completeness and compliance for documentation of procedures in a large, tertiary, teaching hospital.

\section{Review of Literature}

The operative record is a documentation of the process that occurred in the operating room and the findings of the surgeon during the procedure, and must be closely evaluated. However, appropriate and accurate operative records has been identified as a significant area of weakness in surgical patient care. ${ }^{1-5}$

Template-based summaries are not new in medicalsurgical practice. Newer documentation methods have been developed that allow for standardized operative reports also known as synoptic operative reports (SR). Attempts were made to replace NR with electronic forms to achieve a more concise format. This evolution allows the institutions to archive essential information with the integration of electronic medical records as the new standard in the United States. ${ }^{6-8}$

In the local setting, the vast majority of daily progress notes, clinical abstracts and discharge summaries are written freehand by the student, resident or fellow-in-charge. This has been found to be time-consuming to the health care providers given the large volume of patients in the charity service. The NRs consist of the procedure narrated by a physician member of the surgical team. It is created freeform and unstructured in format and content.
At present, the NR contains large amounts of nonessential information while frequently omitting important data. Details such as 'abdomen was prepped and draped' and 'wound was closed in three layers' have little reporting value. In addition, a long narrative report with non-essential details that obscure information makes it difficult to read and burdensome to use in research analysis.

SRs are the expected evolution of this trend. A review of the literature showed that synoptic reports can be successfully created for multiple standardized operative procedures. ${ }^{9}$ It offers several advantages over traditional operative record; it is easier to create and interpret, and more conducive to data extraction for research purposes and outcome analysis. Evidence shows that the synoptic version in general surgery procedures are more complete and have been effectively applied in thyroidectomies, breast surgery, laparoscopic cholecystectomy. ${ }^{5}$ From 2001 to 2003, a provincial Cancer Surgery Working Group led by Edhemovic, et al., designed and piloted a SR in rectal cancer surgery to replace the NR. This included a precise description of the procedure, data on demographics, diagnostic evaluation, staging, and functional measures. A total of 70 items for anterior resection and 63 items for abdominoperineal excision were included. Results showed that NR contained $45.9 \%$ of the specified data elements while the SR captured 99\%. The time needed to complete the SR was only 6 minutes. ${ }^{5} \mathrm{~A}$ prospective study done by Gur, et al., compared 60 consecutive breast cancer narrative operative reports, dictated by the attending surgeon versus the synoptic computerized operative reports filled by an operating resident for overall completeness and for the completeness of individual items. Results showed that the SR contained $94.7 \%$ of the preoperative and operative data, whereas the dictated operative report was able to capture only $66 \%$ of the data $(P<.001){ }^{3}$ A prospective multicenter electronic web-based form was designed and implemented by Iyer, et al. for thyroid surgery from 2008 to 2009. During the study period, 514 synoptic forms from 384 total thyroidectomies and 130 thyroid lobectomies had $100 \%$ compliance. All users found the form to be easy to use, comprehensive, and took less than 5 min to complete. ${ }^{10}$ Studies on surgeries for gynecology using synoptics, however, are limited and is not well-established.

\section{Significance of the study}

This study aims to prove that the electronic synoptic record for operative techniques and findings is superior than the traditional narrative operative record in gynecologic surgery. This can be used as a potential tool to collect concise and comprehensive operative information, requiring minimum time commitment from the surgeon and allowing database storage for use by other subspecialties training in the institution. This tool has the potential to facilitate clinical trials and improve communication between investigators by standardizing and precisely describing operating procedures and intraoperative findings. 


\section{OBJECTIVE}

\section{General Objective}

1. To compare the completeness of NRs and SRs on ovarian, fallopian, and peritoneal cancer surgery

\section{Specific Objectives}

1. To compare the length of time to accomplish the NR and the SR

2. To determine the ease of use of the SR

\section{METHODS}

\section{Study Design, Setting and Duration}

This was a comparative, cross-sectional study conducted at the University of the Philippines-Philippine General Hospital, a tertiary teaching hospital in Manila, Philippines from August to November 2019.

\section{Sampling Design and Sample Size Calculation}

Sample size was calculated using the total number of surgeries done per month for ovarian malignancies (12.73 per month) (2018 Annual Department Census). Since the study duration was 4 months, the population size for the 4-month surgical cases was computed to be 63.75 cases. Using OpenEpi version 3, the calculated sample size, with a $95 \%$ confidence interval for the duration of 4 months was 55 (Table 1).

\section{Eligibility Criteria}

\section{Inclusion Criteria}

1. All cases of probable ovarian, fallopian tube or peritoneal malignancy that were scheduled for operation by the gynecology resident

2. All cases preoperatively referred to the Gynecologic Oncology service for intraoperative surgical staging and/or debulking

\section{Exclusion Criteria}

1. Cases with late stage disease in which only minilaparotomy or biopsy was done or the planned procedure was not completed

2. Cases of probably ovarian malignancy in which the fellow-in-training was the primary surgeon

3. Ovarian malignancy cases that were for interval debulking surgery

\section{Operational Definition of Terms}

Completeness. Presence of previously established quality indicators in the operative reports

Narrative operative report (NR). A handwritten or typewritten documentation of the operative procedure and the findings of the surgeon during the procedure

Synoptic operative report (SR). An electronic format of a procedure-specific, template-based and standardized record of the procedure and findings in the operation

\section{Data Collection}

The cases were selected pre-operatively according to the OR schedule submitted by the resident-on-duty. The chief fellow of the Section of Gynecologic Oncology assessed all cases of probable ovarian, peritoneal or tubal malignancy one day prior to the scheduled procedure. An informed consent was obtained from the resident who was scheduled to perform the procedure and fellows-in-training who would scrub in the case.

The procedure was done by general gynecologic residents or by a gynecologic oncology fellow-in-training. On the day of surgery, the resident accomplished the NR and attached it to the chart prior to transferring the patient to the recovery room. The fellows who assisted the residents in the same surgery accomplished the SR after the procedure in an electronic form which was pre-installed in the operating room (Appendices 1 and 2). A printer was installed in the operating room so that the fellows and residents were able to attach the reports to patient chart prior

Table 1. Sample size computation

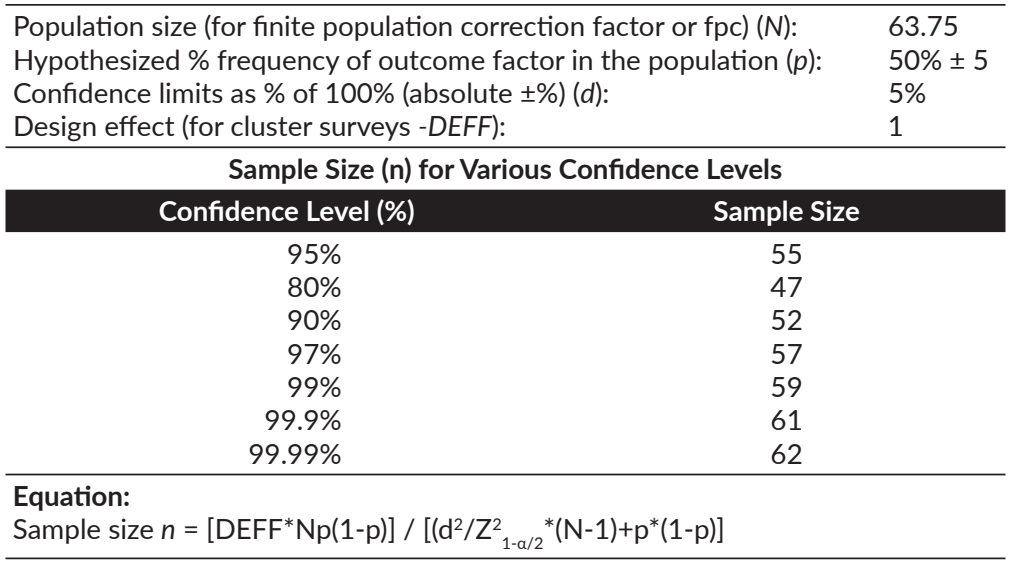

Results from OpenEpi, Version 3, open source calculator - SSPropor 
to transfer to recovery room. The time of completion from the start of writing and the time required to accomplish both reports were recorded by the investigator.

The NRs submitted by the resident surgeon were evaluated for completeness using the Canadian Structured Assessment Format for Evaluating Operative Reports $(\mathrm{SAFE}-\mathrm{OR})^{11}$ The resident surgeons were not aware of the content of the synoptic report to avoid data collection bias. We used the synoptic version tool from the Surgical Manual of the Korean Gynecologic Oncology Group for ovarian, tubal, and peritoneal cancers in this study. The surgical procedures derived from the manual was the first version used by the group to accommodate various clinical trials and represented the minimum requirements for ovarian, peritoneal and tubal cancers with the primary objective of surgical staging. This tool was established on the basis of the Synoptic Operative Template for Ovarian Cancer of National Cancer Center of Korea and was used as the standardized operative record form to document all required information and surgical procedures. ${ }^{12}$ By using this version of synoptic tool, investigators were able to identify all procedures, making the tool the gold standard to standardize operative reports for ovarian, tubal and peritoneal cancer surgeries. Since the tool was used for the first time in the OR setting, the ease of its use was also evaluated and served as a pilot test of the tool. At the end of the duration of this study and upon completion of the data collection, the fellows who used the SR answered a questionnaire to gather comments and evaluate the tool used to accomplish the SR. The participants accomplished the questionnaires after submitting the SR or within 24 hours from the time of submission of the reports. The time to accomplishment of the questionnaires took less than 5 minutes. Study flow chart is shown in Figure 1.

\section{Ease of Use of Synoptic Reports}

At the end of the study, the fellow surgeons completed a survey to assess the ease of use of the synaptic operative reports and their perceptions on the implementation of the synoptic operative reporting system.

This checklist included 65 items, which were recorded as dichotomous variables: ' 1 ' for those described in the

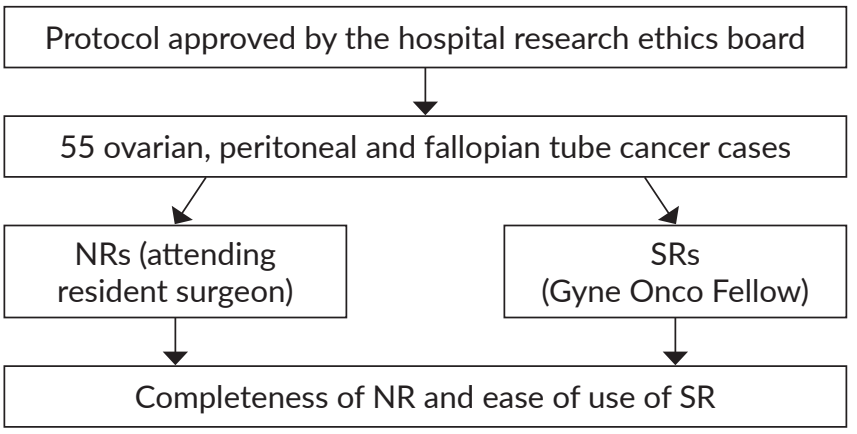

Figure 1. Study flow chart. report, '0' for items absent from the report, or 'N/A' for elements not applicable to the case. Percent and total item completion scores were tabulated for all reports excluding 'N/A' items from the total. We compared percent and total completeness scores between the NR and SR cohorts and computed for difference of means using paired-samples t-tests with a two-tailed alpha. Statistical significance was set at an alpha of 0.05 .

\section{Statistical Analysis}

We encoded the information from the NRs in a binary fashion using Microsoft Excel: 1 (present), 0 (absent), NA (nonapplicable). Individual items and overall completeness of traditional narrative and synoptic operative reports were compared using Fisher's exact test. A P value of 0.05 or less was considered statistically significant. T-test was performed for the comparison of the time need to accomplish the NR and SR.

\section{Ethical Considerations}

Upon approval by the hospital research ethics board, an informed consent was obtained from the residents and fellows prior to commencement of data collection. All case registry forms were kept in the investigator's locked cabinet and computers used were password-protected. There were no human participants involved since only NRs and SRs made by the surgeons were collected.

\section{RESULTS}

There were $55 \mathrm{NRs}$ and SRs included in the study. Among the cases were 53 ovarian, 1 peritoneal and 1 tubal malignancy for primary surgical staging and tumor debulking performed from August to November 2019.

The overall percentage of reported quality indicators was $77.1 \%$ (average, $35.7 / 65$ ), while 10.6 was the average number of absent indicators (Table 2).

Table 2. Overall completeness of quality indicators

\begin{tabular}{lcc} 
& Mean & SD \\
Number of Reported Indicators & 35.7 & 2.3 \\
Number of Unreported Indicators & 10.6 & 2.2 \\
Number of Not Applicable & 18.6 & 2.4 \\
Average Estimated \% of Present Indicators & $77.1 \%$ & \\
\hline
\end{tabular}

A total of 27 indicators were completely present in all 55 forms, including date of procedure, patient identifier, age, and $\mathrm{OB}$ score. On the other hand, 8 quality indicators were completely absent in the 55 forms; namely, the anesthesiologist, planned procedure, thromboprophylaxis, compression stockings, antibiotic prophylaxis, preoperative tumor markers, operative time and report date. The most poorly reported indicator was the presence or absence of residual lesions (29.1\%) (Table 3). 
Table 3. Completeness of quality indicators

\begin{tabular}{|c|c|c|c|c|}
\hline & Number of Present & $\%$ Completeness & $95 \% \mathrm{Cl}$ & Rank \\
\hline Date of procedure & 55 & 100.0 & 93.5 to 100 & 1 \\
\hline Patient identifier & 55 & 100.0 & 93.5 to 100 & 1 \\
\hline Age & 55 & 100.0 & 93.5 to 100 & 1 \\
\hline OB score & 55 & 100.0 & 93.5 to 100 & 1 \\
\hline Comorbidities & 54 & 98.2 & 90.4 to 99.7 & 28 \\
\hline Other gynecologic conditions & 51 & 92.7 & 82.7 to 97.1 & 36 \\
\hline Previous abdominal surgeries & 50 & 90.9 & 80.4 to 96.1 & 38 \\
\hline Attending surgeon & 55 & 100.0 & 93.5 to 100 & 1 \\
\hline $1^{\text {st }}$ Assistant & 54 & 98.2 & 90.4 to 99.7 & 28 \\
\hline $2^{\text {nd }}$ assistant & 53 & 96.4 & 87.7 to 99.0 & 33 \\
\hline Anesthesia & 53 & 96.4 & 87.7 to 99.0 & 33 \\
\hline Anesthesiologist & 0 & 0.0 & 0.0 to 6.5 & 52 \\
\hline Pre-op diagnosis & 55 & 100.0 & 93.5 to 100 & 1 \\
\hline Procedure planned & 0 & 0.0 & 0.0 to 6.5 & 52 \\
\hline Procedure performed & 55 & 100.0 & 93.5 to 100 & 1 \\
\hline Post-op diagnosis & 55 & 100.0 & 93.5 to 100 & 1 \\
\hline Thromboprophylaxis & 0 & 0.0 & 0.0 to 6.5 & 52 \\
\hline Compression stockings & 0 & 0.0 & 0.0 to 6.5 & 52 \\
\hline Skin preparation type & 36 & 65.5 & 52.3 to 76.6 & 47 \\
\hline Draping & 55 & 100.0 & 93.5 to 100 & 1 \\
\hline Antibiotic prophylaxis & 0 & 0.0 & 0.0 to 6.5 & 52 \\
\hline FIGO staging & 49 & 89.1 & 78.2 to 94.9 & 39 \\
\hline Primary site & 55 & 100.0 & 93.5 to 100 & 1 \\
\hline Preoperative tumor markers & 0 & 0.0 & 0.0 to 6.5 & 52 \\
\hline Patient position & 55 & 100.0 & 93.5 to 100 & 1 \\
\hline Drape & 55 & 100.0 & 93.5 to 100 & 1 \\
\hline Incision & 55 & 100.0 & 93.5 to 100 & 1 \\
\hline Fertility preservation & 55 & 100.0 & 93.5 to 100 & 1 \\
\hline Type of hysterectomy & 55 & 100.0 & 93.5 to 100 & 1 \\
\hline Type of vault closure & 55 & 100.0 & 93.5 to 100 & 1 \\
\hline Salpingo-oophorectomy & 55 & 100.0 & 93.5 to 100 & 1 \\
\hline Peritonectomy & & Not Applicable & & \\
\hline Omentectomy & 55 & 100.0 & 93.5 to 100 & 1 \\
\hline Appendectomy & 49 & 89.1 & 78.2 to 94.9 & 39 \\
\hline Bowel resection & & Not Applicable & & \\
\hline Splenectomy & & Not Applicable & & \\
\hline Other organ resection & 54 & 98.2 & 90.4 to 99.7 & 28 \\
\hline Level of lymphadenectomy & 54 & 98.2 & 90.4 to 99.7 & 28 \\
\hline Debulking site & 55 & 100.0 & 93.5 to 100 & 1 \\
\hline Other procedure & 44 & 80.0 & 67.6 to 88.5 & 44 \\
\hline Intraoperative findings & 55 & 100.0 & 93.5 to 100 & 1 \\
\hline Frozen section & 55 & 100.0 & 93.5 to 100 & 1 \\
\hline Ascites & 53 & 96.4 & 87.7 to 99.0 & 33 \\
\hline Adhesions & 47 & 85.5 & 73.8 to 92.4 & 42 \\
\hline Ovarian tumor size & 55 & 100.0 & 93.5 to 100 & 1 \\
\hline Residual lesions & 16 & 29.1 & 18.8 to 42.1 & 50 \\
\hline Lymph node enlargement & 49 & 89.1 & 78.2 to 94.9 & 39 \\
\hline Intraperitoneal tumor & & Not Applicable & & \\
\hline Extraperitoneal tumor & & Not Applicable & & \\
\hline Anti-adhesive used & & Not Applicable & & \\
\hline Hemostatic agent used & 51 & 92.7 & 82.7 to 97.1 & 36 \\
\hline Intraoperative injury & 1 & 50.0 & 9.5 to 90.6 & 51 \\
\hline Estimated blood loss & 54 & 98.2 & 90.4 to 99.7 & 28 \\
\hline Blood transfusion & 40 & 72.7 & 59.8 to 82.7 & 46 \\
\hline Drains used & 47 & 85.5 & 73.8 to 92.4 & 42 \\
\hline Location of drain & 43 & 78.2 & 65.6 to 87.1 & 45 \\
\hline Gauze count & 55 & 100.0 & 93.5 to 100 & 1 \\
\hline Type of ligating suture & 55 & 100.0 & 93.5 to 100 & 1 \\
\hline Wound closure, type of suture & 55 & 100.0 & 93.5 to 100 & 1 \\
\hline Wound closure, type of stitches & 55 & 100.0 & 93.5 to 100 & 1 \\
\hline List of specimens sent & 30 & 54.5 & 41.5 to 67.0 & 49 \\
\hline Operative time (hr:min) & 0 & 0.0 & 0.0 to 6.5 & 52 \\
\hline Post-operative condition & 32 & 58.2 & 45.0 to 70.3 & 48 \\
\hline Signature of surgeon & 55 & 100.0 & 93.5 to 100 & 1 \\
\hline Report date & 0 & 0.0 & 0.0 to 6.5 & 52 \\
\hline
\end{tabular}


The mean time to accomplish the SR (10.4 minutes, $\mathrm{SD} 4.3$, range 6 to 25) was significantly shorter than the mean time it took to accomplish the NR (21.9 minutes, SD 10.5 , range 9 to 66 ) by 11.5 minutes ( $95 \%$ CI 8.3 to 14.6 ) $(\mathrm{p}=0.0001)$ (Figure 2).

A wrong post-operative diagnosis was reported in $12.7 \%$ while $7.3 \%$ of reports had the wrong mode of anesthesia. In the operative technique, the most common errors were: missed procedures $(27.3 \%)$, discrepancy in reported skin suture $(22.6 \%)$ and wrong laterality of adnexa that was removed (16.4\%). The most common missing intra-operative findings were: description of the bladder (65.6\%), followed by stomach (27.3\%) and omentum (21.8\%) (Table 4).

\section{Ease of use of SRs}

\section{Timeliness of NRs}

Majority $(71 \%, 5 / 7)$ of the gynecologic oncology fellowsin-training reported excessive delays in the timeliness of the NRs that were received for gynecologic malignancy surgeries while the others reported only short delays in the reports. Among the NRs submitted by the resident surgeons, three surgeons were unable to comply with the submission of 10 NRs prior to transfer of these patients to the recovery room and was able to place the NR on the $2^{\text {nd }}$ day post op.

\section{Experience with poorly written operative reports}

All gynecologic oncology fellows experienced a situation where poor operative reports have led to poor patient care, such as: residual tumor not reported, rupture of the mass not indicated, inconsistencies with the actual operative findings, incorrect labels, wrong laterality. These resulted in delay in treatment planning and initiation of treatment.

\section{Improving the NRs}

On how to improve reporting of the NRs, the following were suggested: ensuring completeness and taking time to finish the reports immediately post-operative upon scrubbing out of the OR, indicating the extent and not just the measurement, listing all surgeons involved especially if with morbidities, and using a typewritten but uniform template from the institution.

\section{Information on SRs}

All but one fellow did not have experience in reading or interpreting a synoptic report.

\section{Superiority of SRs to NRs}

All except one fellow strongly agreed that SRs are superior to NRs in several areas of surgery for quality, completeness and timeliness. All except one agreed that a SR could improve the operative reports in gynecologic surgery.

\section{General comments on SRs}

The comments on the SR used in this study includes the following: convenience since it is accomplished right after the surgery, standardization of reports, ease of use except that there must be room for unusual findings. The SR was useful and helpful and it was hoped to become the standard in the future.

\section{Key features for an ideal SR}

An ideal SR for gynecologic surgery would have the following key features: simple format, time-efficient, easy to understand and follow.

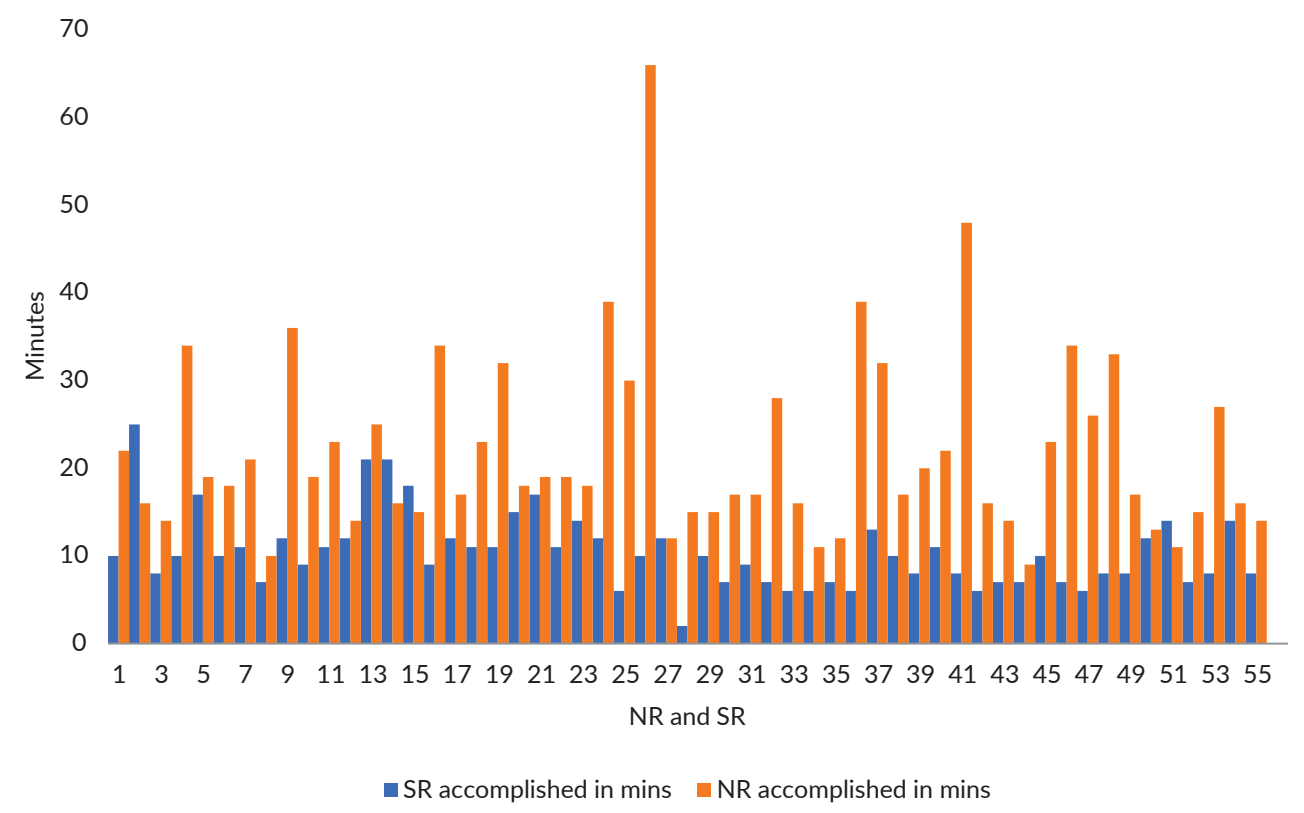

Figure 2. Time required to accomplish the narrative reports and synoptic reports. 
Table 4. Errors or omissions in narrative reports

\begin{tabular}{|c|c|c|}
\hline Error or omission & $\mathbf{N}$ & $\%$ \\
\hline \multicolumn{3}{|l|}{ General Information } \\
\hline Incorrect post-operative diagnosis & 7 & 12.7 \\
\hline Name of the $2^{\text {nd }}$ assist is incorrect & 1 & 1.8 \\
\hline 2nd assist not mentioned & 3 & 5.5 \\
\hline Random peritoneal biopsy is missing in procedure done & 1 & 1.8 \\
\hline Case number missing & 1 & 1.8 \\
\hline Jackson-Pratt drain in procedure not mentioned & 2 & 3.6 \\
\hline Wrong mode of anesthesia & 4 & 7.3 \\
\hline Previous surgery not mentioned & 3 & 5.5 \\
\hline Wrong spelling in diagnosis & 1 & 1.8 \\
\hline Procedure missed & 7 & 12.7 \\
\hline \multicolumn{3}{|l|}{ Operative Technique } \\
\hline Extent of abdominal incision not mentioned & 3 & 5.5 \\
\hline Infracolic omentectomy not mentioned among the steps & 2 & 3.6 \\
\hline Peritoneal fluid cytology not mentioned among the steps & 2 & 3.6 \\
\hline Wrong laterality of adnexa removed & 9 & 16.4 \\
\hline Suture used in skin is different & 8 & 14.5 \\
\hline Suture used in subcutaneous tissue is different & 8 & 14.5 \\
\hline Missing procedure & 15 & 27.3 \\
\hline Foley catheter inserted in endocervical canal was stated but not actually done & 4 & 7.3 \\
\hline Ligation of contralateral adnexa was not mentioned & 5 & 9.1 \\
\hline Redundant steps & 2 & 3.6 \\
\hline \multicolumn{3}{|l|}{ Intra-operative Findings } \\
\hline Bladder & 36 & 65.5 \\
\hline Stomach & 15 & 27.3 \\
\hline Liver & & 5.5 \\
\hline Subdiaphragm/peritoneum & & 12.7 \\
\hline Gallbladder & & 16.4 \\
\hline Omentum & & 21.8 \\
\hline Spleen & & 7.3 \\
\hline Kidneys & & 12.7 \\
\hline Intestines & & 16.4 \\
\hline Appendix & & 7.3 \\
\hline Uterus & & 7.3 \\
\hline Cervix & & 1.8 \\
\hline Myometrium & & 1.8 \\
\hline Contralateral adnexa & & 14.5 \\
\hline Uterine cavity & & 10.9 \\
\hline Endocervical canal & & 10.9 \\
\hline Pelvic lymph node & & 9.1 \\
\hline Paraaortic lymph node & & 14.5 \\
\hline Adhesions & & 14.5 \\
\hline Ascites & 2 & 3.6 \\
\hline Serosal tear & 1 & 1.8 \\
\hline Inferior vena cava injury & 1 & 1.8 \\
\hline
\end{tabular}

\section{DISCUSSION}

The study highlights the need for robust operative note recording practices and surgeons need to be well trained for this. All 55 NRs lacked specific quality indicators needed for safe operating room practices. The incorrect mode of anesthesia, incorrect post-operative diagnosis and missing procedures in the general data will result in problems regarding reimbursement procedures due to conflicting data in the NR. This will lead to patient inconvenience and cause stress. It is time-consuming to look for the surgeon to sign the corrections in the NRs. Mentioning a wrong procedure, such as removal of the adnexa with wrong laterality, may also lead to medicolegal problems and litigations. Four NRs mentioned insertion of a small caliber Foley catheter in the endocervical canal which is usually done in patients who will undergo chromotubation. This reflects the practice of the surgeon 'copy-paste' a previous NR without double checking the statements. This also contributes to the errors made in the NRs. In addition, most NRs failed to mention complete intraoperative findings. This may be attributed to poor recall, hence the need to accomplish these forms immediately after the procedure. It may also suggest poor assessment of different abdominopelvic organs; either the abdominopelvic organs were fully assessed or not, hence, the failure to mention. This will significantly affect patient post-operative care, especially in patients with residual tumors. Mentioning the presence 
of any residual tumors will be of value to gynecologic oncologists for post-operative follow-up, planning adjuvant treatment, monitoring and prognosis of patients with ovarian, peritoneal and tubal malignancies.

The study results point to a need for better education programs, mentorship by seniors and a quality assurance process. Formal teaching regarding operative notes is a must in modern-day surgical practice. Quality operation note recording facilitates quality medical care, provides defense from litigation, and remuneration for surgical procedures. In gynecologic surgery, our data suggest that training on the completion of the NRs has been mostly ignored in surgical training. Our study presents a local perspective of residents' and fellows' perceptions about the current status of operative report training, which represents an important but poorly studied area with significant clinical and educational implications. The findings of our study have certain limitations in that the time for data collection was limited to 4 months. However, the findings serve as a needs assessment, to identify deficiencies in current NRs and to guide formal training in NR skills through regular feedback and operative finding templates.

The excessive delays in the reporting of the NRs, up to the $2^{\text {nd }}$ post-operative day, may lead to problems in recall especially for the senior residents who do the bulk of procedures. Further studies are recommended to investigate the factors that led to this delay and how to further improve the timeliness of reporting. The lack of computers and printer in the OR area may have also contributed to delays in insertion of the NRs into the charts. With increasing subspecialization in gynecologic surgeries, there is a wide array of surgical operations that include a multitude of technologies. This means greater demands from operation record for clinical care, medico-legal purposes and clinical coding in the future.

\section{CONCLUSION}

Incompleteness and delays in accomplishing NRs for patients post-surgery for ovarian, fallopian tube, and peritoneal cancers, can be improved with the use of more accurate SRs. Synoptic reports are easier to use and faster to complete, provided that computers are readily available in operating theatres, and users are adequately trained. An added advantage is that the data collected may be archived and analyzed automatically for audit and research. Periodic audit is suggested to assure timely reporting. Formal teaching sessions in writing operative records will be helpful to improve the quality of operative recording.

\section{Statement of Authorship}

Both authors participated in data analysis, and approved the final version submitted.

\section{Author Disclosure}

Both authors declared no conflicts of interest.

\section{Funding Source}

This paper was funded by the UP-PGH Research Grant Award 2019.

\section{REFERENCES}

1. Nicopoullos JDM, Karrar S, Gour A, Panter K. Significant improvement in quality of caesarean section documentation with dedicated operative proforma - Completion of the audit cycle. J Obstet Gynaecol. 2003 Jul; 23(4):381-6. doi: 10.1080/0144361031000119547.

2. Harvey A, Zhang H, Nixon J, Brown CJ. Comparison of data extraction from standardized versus traditional narrative operative reports for database-related research and quality control. Surgery. 2007 Jun; 141(6):708-14. doi: 10.1016/j.surg.2007.01.022.

3. Gur I, Gur D, Recabaren JA. The computerized synoptic operative report: a novel tool in surgical residency education. Arch Surg. 2012 Jan; 147(1):71-4. doi: 10.1001/archsurg.2011.228.

4. Gardner GJ, Leitao MM, Mitchell M, Levine DA, Brown CL, Sonoda Y, et al. Prototype of a synoptic electronic operative note for gynecologic oncology surgical procedures. Gynecol Oncol. 2009 Feb; 112(2 Suppl):S64-S65.

5. Edhemovic I, Temple WJ, de Gara CJ, Stuart GCE. The computer synoptic operative report-- a leap forward in the science of surgery. Ann Surg Oncol. 2004 Oct; 11:941-7. doi: 10.1245/ASO. 2004.12.045.

6. Lissauer T, Paterson CM, Simons A, Beard RW. Evaluation of computer-generated neonatal discharge summaries. Arch Dis Child. 1991 Apr; 66(4 Spec No.):433- 6. doi: 10.1136/adc.66.4_spec_ no.433.

7. van Walraven C, Laupacis A, Seth R, Wells G. Dictated versus database- generated discharge summaries: a randomized clinical trial. CMAJ. 1999 Feb; 160(3):319-26.

8. Adams DC, Bristol JB, Poskitt KR. Surgical discharge summaries: improving the record. Ann R Coll Surg Engl. 1993 Mar; 75(2):96-9.

9. Chambers AJ, Pasieka JL, Temple WJ. Improvement in the accuracy of reporting key prognostic and anatomic findings during thyroidectomy by using a novel web-based synoptic operative reporting system. Surgery. 2009 Dec; 146(6):1090-8. doi: 10.1016/ j.surg.2009.09.032.

10. Iyer NG, Nixon IJ, Palmer F, Ganly I, Patel SG, Shaha AR. Electronic synoptic operative reporting for thyroid surgery using an electronic data management system: potential for prospective multicenter data collection. Ann Surg Oncol. 2011 Mar; 18(3):762-6. doi: 10.1245/ s10434-010-1361-0.

11. Vergis A, Gillman L, Minor S, Taylor M, Park J. Structured assessment format for evaluating operative reports in general surgery. Am J Surg. 2008 Jan; 195(1):24-9. doi: 10.1016/j.amjsurg.2007.08.053.

12. Jeon S, Lee SJ, Lim MC, Song T, Bae J, Kim K, et al. Surgical manual of the Korean Gynecologic Oncology Group: ovarian, tubal, and peritoneal cancers. J Gynecol Oncol. 2017 Jan; 28(1):e6. doi: 10.3802/jgo.2017.28.e6. 


\section{APPENDICES}

Appendix 1. Actual Synoptic Electronic Form Used for Gynecologic Surgery
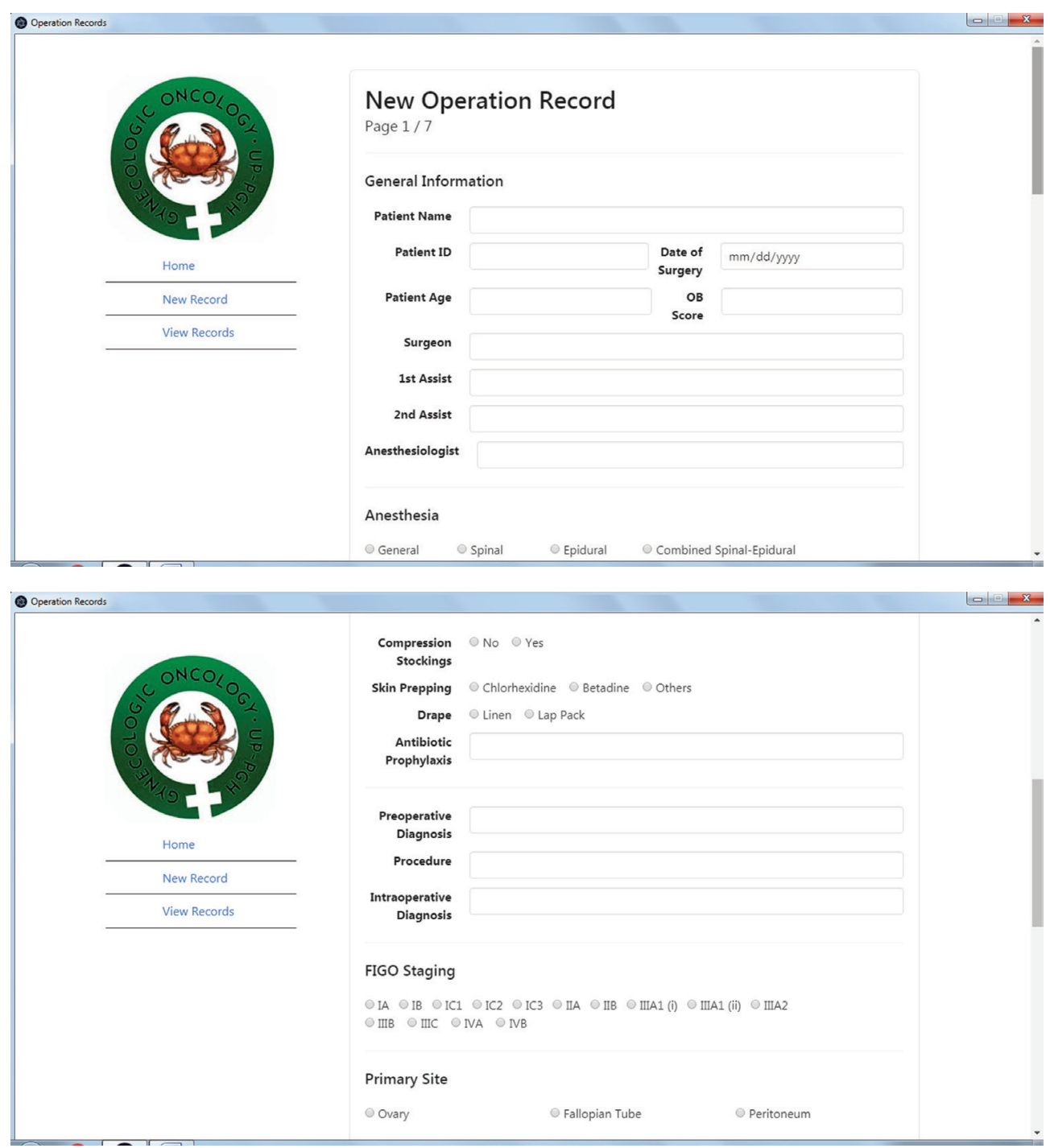

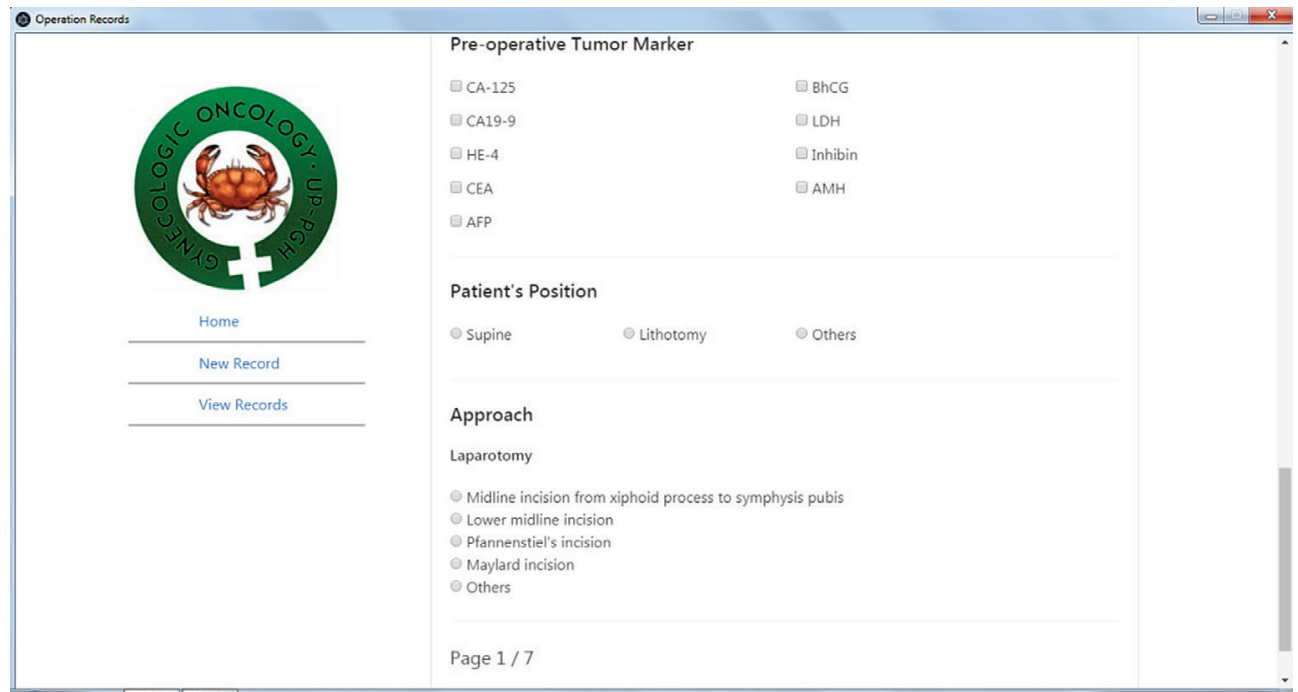


Use of Synoptic Operative Report for Gynecologic Surgery
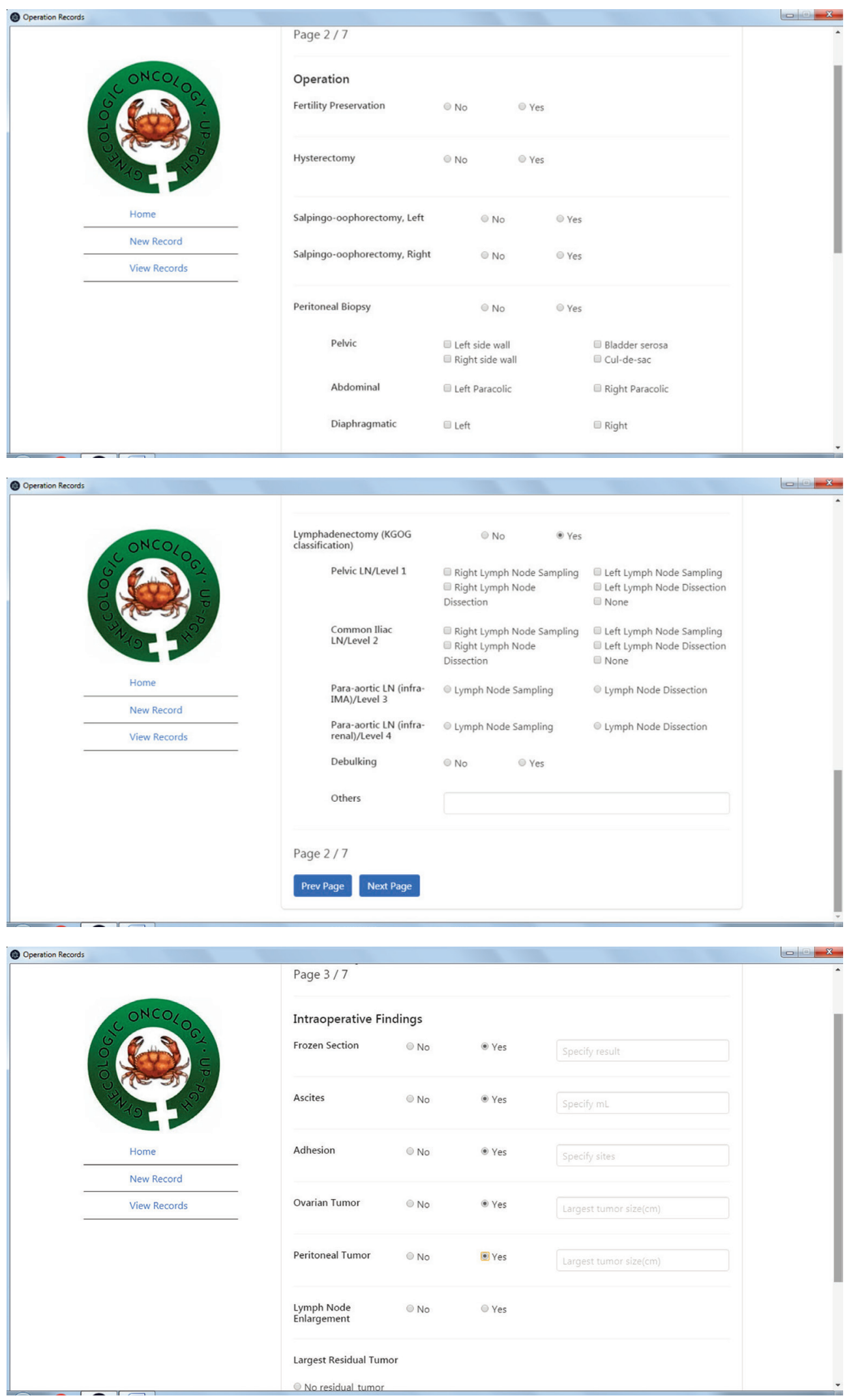


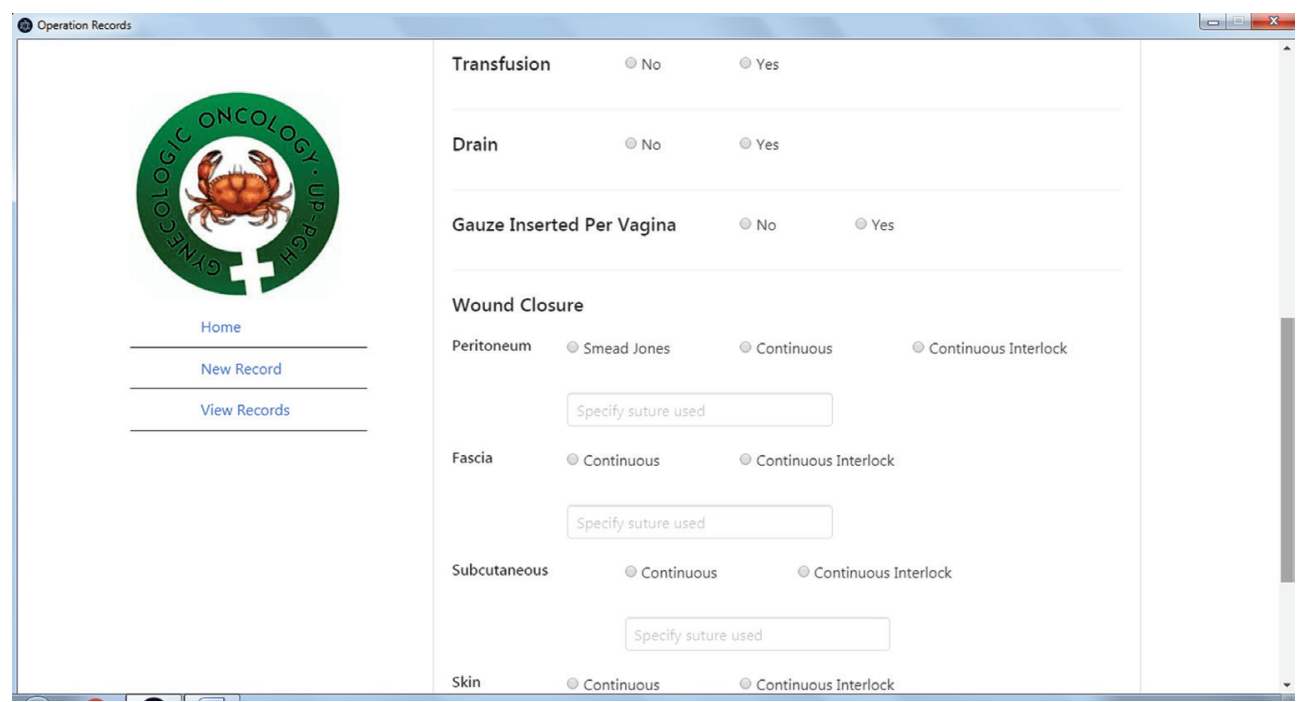

Appendix 2. Sample of Synoptic Report after Gynecologic Surgery

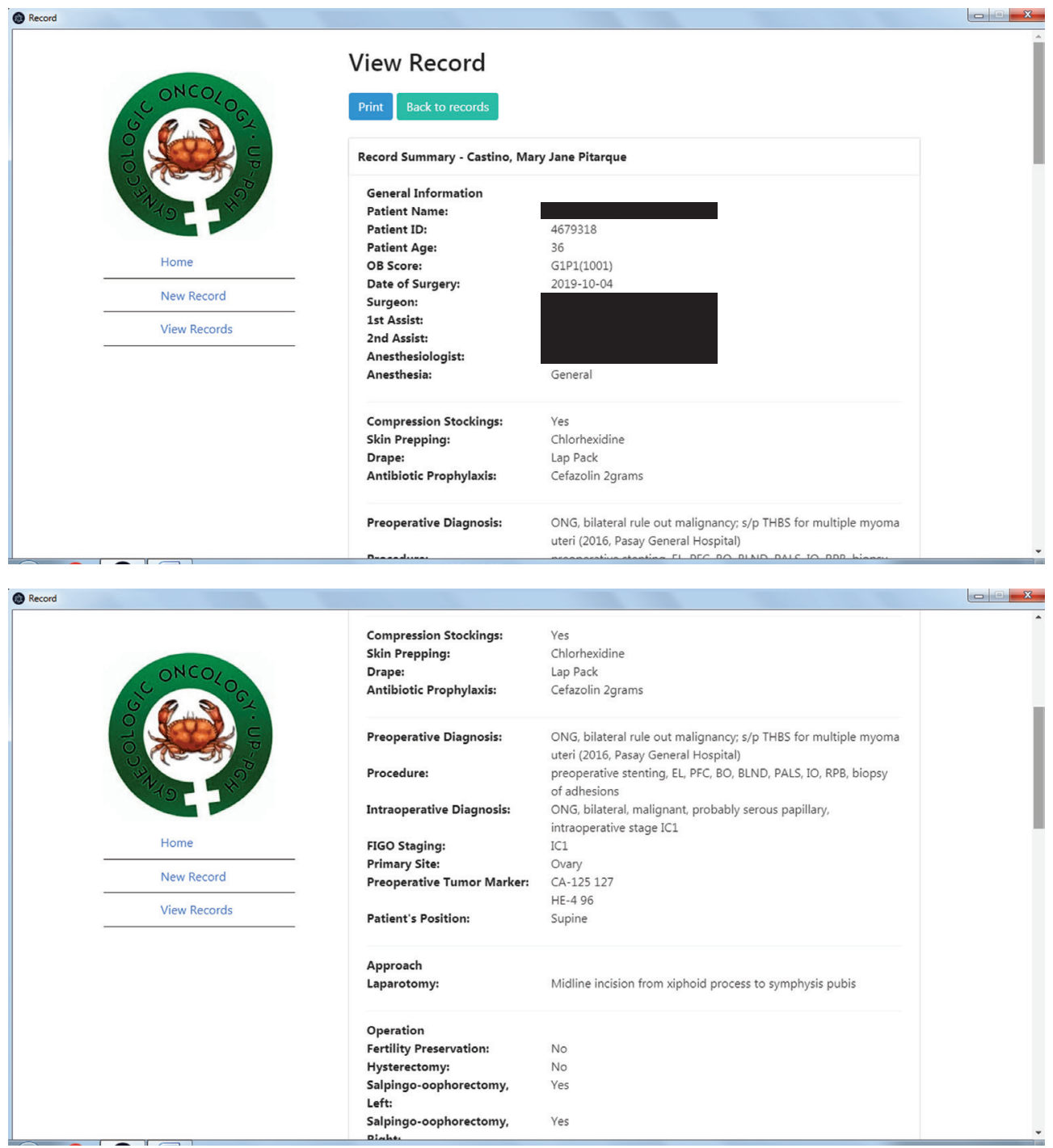


Use of Synoptic Operative Report for Gynecologic Surgery

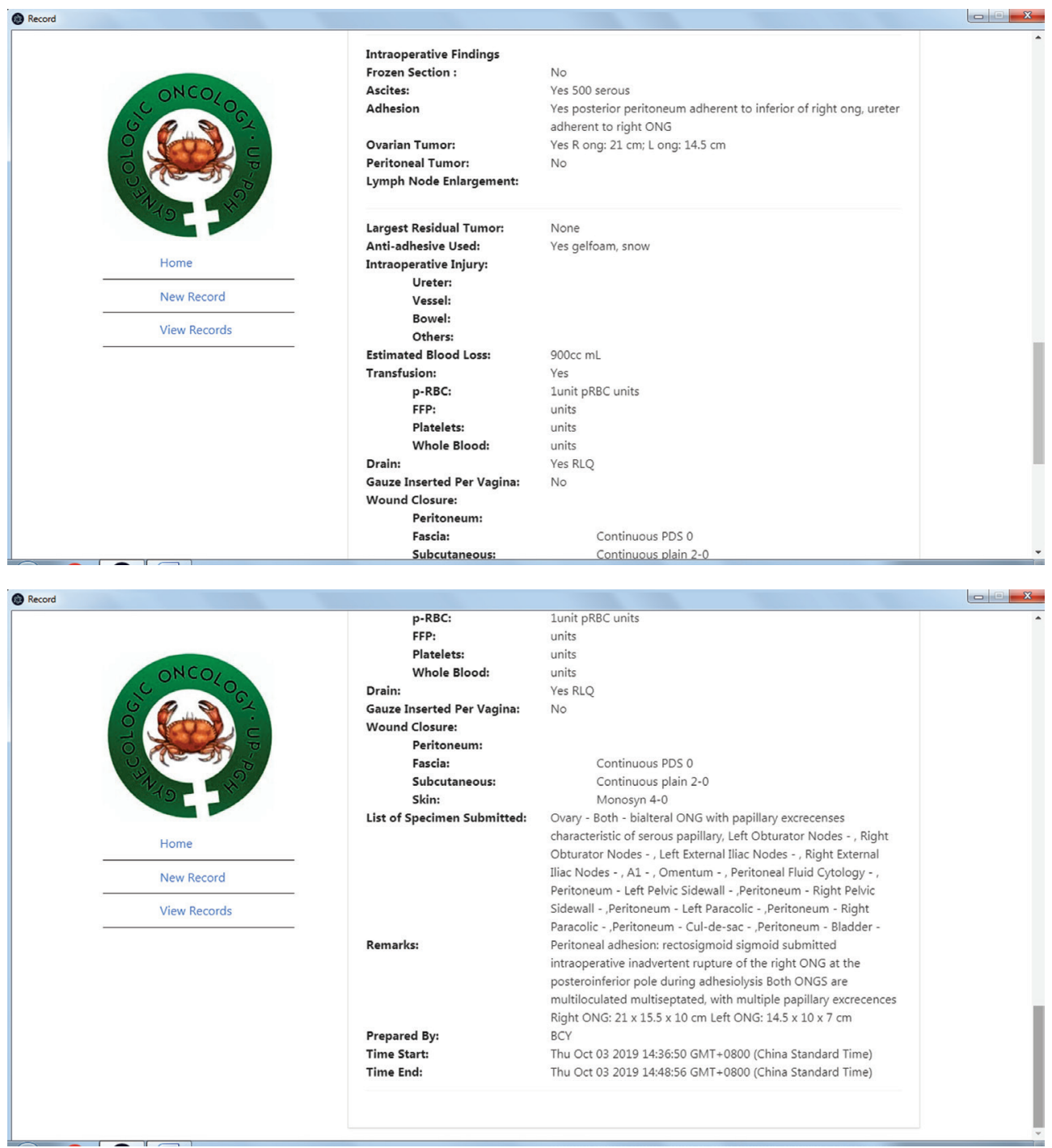

